\title{
Non-antibiotic approaches at drying-off for treating and preventing intramammary infections: a protocol for a systematic review and meta-analysis
}

\author{
David Francoz ${ }^{1}$, Vincent Wellemans ${ }^{1}$, Jean-Philippe Roy ${ }^{1,2}$, Pierre Lacasse $^{2,3}$, \\ Alfredo Ordonez-Iturriaga ${ }^{1}$, François Labelle ${ }^{4}$ and Simon Dufour ${ }^{2,5 *}$ \\ ${ }^{1}$ Département de sciences cliniques, Faculté de médecine vétérinaire, Université de Montréal, \\ C.P. 5000, St-Hyacinthe, QC, J2S 7C6, Canada \\ ${ }^{2}$ Canadian Bovine Mastitis and Milk Quality Research Network, C.P. 5000, St-Hyacinthe, QC, J2S \\ 7C6, Canada \\ ${ }^{3}$ Sherbrooke Research and Development Centre, Agriculture and Agri-Food Canada, 2000 \\ Collège, Sherbrooke, QC, J1M 0C8, Canada \\ ${ }^{4}$ Département de pathologie et microbiologie, Faculté de médecine vétérinaire, Université de \\ Montréal, C.P. 5000, St-Hyacinthe, QC, J2S 7C6, Canada \\ ${ }^{5}$ Valacta, 555 Boul. des Anciens-Combattants, Ste-Anne-de-Bellevue, QC, H9X 3R4, Canada
}

Received 24 May 2016; Accepted 9 September 2016

\begin{abstract}
Intramammary infection (IMI) treatment and prevention at drying-off is one of the leading causes for using antimicrobials on dairy farms. The objective of the current paper is to describe the protocol used for conducting a systematic review of the literature on non-antibiotic strategies that can be used on dairy cows at dry off to treat and prevent IMI. Relevant literature will be identified using a combination of database search strategies and iterative screening of references. To be included in the review, articles will have to: (1) be published after 1969; (2) be written in English, French, or Spanish; (3) use a study design such as a controlled trial, an observational study, or an experimental study conducted in vivo; (4) be conducted on commercial dairy cows; (5) investigate a non-antibiotic intervention used at dry off; and finally, (6) report on a relevant mastitis outcome. Titles and abstracts, then full articles will be reviewed for inclusion. Specific data will be extracted and risk of bias will be assessed for all included articles. The planned systematic review will be the first to colligate, in a coherent whole, studies investigating non-antibiotic strategies for treating and preventing IMI at drying-off.
\end{abstract}

Keywords: dairy cows, intramammary infection, dry off, treatment, systematic review.

\section{Introduction}

Mastitis is an inflammation of the mammary gland most often caused by intramammary infections (IMI). Mastitis treatment is the most important cause of antimicrobial use in dairies in many countries (Saini et al., 2012; Kuipers et al., 2016).

*Corresponding author. E-mail: simon.dufour@umontreal.ca
At drying-off, for instance, infusion of all quarters with an antibiotic is a common practice. This practice is usually used for treating existing IMI and for preventing acquisition of new IMI, which have been shown to be very frequent at beginning and end of the dry period (Bradley and Green, 2000, 2004).

Numerous non-antimicrobial approaches for clinical mastitis (CM) treatment or for control of IMI, during the lactation or at drying-off, have been investigated, including anti-inflammatory drugs, teat sealant, homeopathic, phytotherapeutic, and 
other biological products, etc. (see Hektoen et al., 2004; Klostermann et al., 2008; Pinedo et al., 2013 for examples). At drying-off, these products can be administered through systemic (i.e. parenteral) or local administration (i.e. intrammammary or external application on the udder). Results on the efficacy of these alternative approaches for treating or preventing IMI at drying-off, however, have not been collated in a coherent whole. Furthermore, the recommendations frequently convey de in the organic dairy sector on the efficacy of some alternative approaches remain questionable and do not appear to be supported by rigorous studies (Mathie and Clausen, 2014, 2015). The objective of the current paper is to describe the protocol used for conducting a systematic review of the literature on non-antibiotic strategies that can be used at dry off to treat or prevent IMI.

\section{Objectives}

The objective of this systematic review will be to identify the strictly non-antibiotic therapeutic strategies for treatment and prevention of IMI at drying-off in dairy cows. Two objectives (i.e. treatment and prevention) are, therefore, pursued and these are described in the following two PICO (Population, Intervention, Comparator, Outcome) questions.

\section{Treating intramammary infections at drying-off}

When compared to intramammary infusion of antibiotic or to no treatment (i.e. the comparators), which strictly non-antibiotic treatment strategies (i.e. the interventions) have been shown to be efficient for improving elimination of IMI (i.e. the outcome) during the dry period in dairy cows (i.e. the population)?

\section{Preventing intramammary infections acquisition during the dry period}

When compared to intramammary infusion of antibiotic and/or to no treatment (i.e. the comparators), which strictly non-antibiotic treatment strategies (i.e. the interventions) have been shown to be efficient for preventing IMI acquisition (i.e. the outcome) during the dry period in dairy cows (i.e. the population)?

\section{Materials and methods}

\section{Eligibility criteria}

\section{Study design}

Studies using designs such as controlled trials (randomized or not) or studies using observational cohort, or case-control designs will be retained. Meta-analyses conducted using studies making use of the aforementioned study designs will also be considered. Cross-sectional study design will not be retained, because main outcomes (see below) cannot be reported using a non-longitudinal design. Less valid study designs such as case-series, case-reports, or expert opinion will be excluded. Moreover, studies conducted in vitro, that cannot support proof of efficacy in the target host, will be excluded.

For studies investigating treatment of existing IMI at dryingoff, studies investigating naturally acquired and/or experimentally induced IMI will be included. For studies investigating IMI prevention, studies using naturally acquired IMI or bacterial challenge using intramammary infusion of bacteria or teat dipping with a solution containing bacteria will be retained.

\section{Population}

Lactating dairy cows at dry off will be the participants of interest. Because extrapolation from other species is difficult, and because of differences in pathogens involved or in immune system function, studies conducted on tropical breeds and other ruminant or non-ruminant species will be excluded. For studies investigating treatment of IMI and reporting IMI elimination rate, the population studied will have to be infected quarters or cows. On the other hand, for studies investigating IMI prevention and reporting on IMI incidence over the dry period, the population studied will be uninfected quarters or cows.

\section{Interventions}

The different non-antibiotic interventions that will be evaluated include all non-antibiotic strategies and treatments used at drying-off, such as anti-inflammatory drugs, teat sealant, as well as homeopathic, phytotherapeutic, or biological products. For all interventions, a treatment group receiving the non-antibiotic treatment solely (versus a group receiving a non-antibiotic treatment combined with an antibiotic) will have to be investigated in the study for it to be included in the current review.

\section{Comparators}

For IMI treatment, because a substantial rate of spontaneous IMI elimination is expected during the dry-off period, only studies comparing the treatment under investigation to a negative (i.e. no treatment or a placebo) and/or to a positive control (i.e. a veterinary compound with demonstrated efficacy for IMI treatment at drying-off) will be included. Similarly, studies investigating IMI prevention will have to make use of either or both a negative and positive control group for inclusion in the current review.

\section{Outcomes}

Outcomes under investigation will vary depending if IMI treatment or prevention is under investigation. For IMI treatment, 
studies reporting on IMI elimination rate over the dry period and measured using one or many pre-dry milk samples and one or many post-calving milk samples and studies reporting on IMI prevalence post-calving using post-calving milk samples solely will be included. Both studies using quarter- or cow-level (i.e. a composite sample of the milk from the four quarters) milk samples will be included. For determination of the quarter or cow IMI status, only studies using the following well-accepted diagnostic tests for IMI definition will be retained: milk somatic cell counts (SCC), routine milk bacteriological culture, or milk polymerase chain reaction (PCR).

For studies investigating IMI prevention, studies reporting incidence rate over the dry period using one or many pre-dry milk samples and one or many post-calving milk samples and, again, studies reporting on IMI prevalence post-calving using only post-calving milk samples will be included. Furthermore, studies reporting on $\mathrm{CM}$ incidence at beginning of following lactation ( $0-3$ months in milk) will also be included since early lactation $\mathrm{CM}$ is a well-documented consequence of dry periodacquired IMI (Bradley and Green, 2000, 2004). Again both studies using quarter- and cow-level sampling will be considered, and only studies using SCC, routine milk bacteriological culture, or PCR for determination of IMI status will be included.

\section{Report characteristics}

In addition to previously mentioned inclusion criteria, to be included, papers will have to: (1) be published after 1969 (to ensure applicability to modern dairies); and (2) be written in English, French, or Spanish. Whenever two or more publications will present results from a same study, only the most complete study will be selected. If needed, authors will be contacted to confirm originality of results from different publications.

\section{Information sources}

Identification of the relevant databases and elaboration of the search strategy were conducted with the help of a librarian. As suggested by Grindlay et al. (2012), two databases, CAB Abstracts and Medline, and one search platform, Web of Science, will be searched on the same day without any language restriction. To complete the database search, whenever a publication will be retained to be included in the systematic review after having undergone the complete selection process (see below), the list of references of the included publication will then be reviewed by the reviewers to identify additional publications not previously identified by the initial database search. These additional publications will then also be evaluated for inclusion by the reviewers as described for the initial electronic search (see below). Whenever this process will result in the inclusion of an additional publication, the list of references of the newly included publication will, again, be screened and the process repeated until complete depletion.

No additional effort will be made, beyond the databases, search platform, and list of references appraisal, to specifically retrieve non-peer-reviewed publications (e.g. asking experts or screening of conference proceedings). This later type of publication will, nevertheless, be retained in the current review when identified by the databases, search platform, or reference screening searches.

\section{Search strategy}

Boolean search strategies using thesaurus terms or natural language used in abstract, title, original title, broad terms, or heading, and based on descriptors of the population of interest (dairy cows), outcome studied (IMI), the treatment aspect (nonantibiotic approach) at a specific period (i.e. dry off period), were designed specifically for each databases and for Web of Science. Regarding the non-antimicrobial treatment aspect, an exhaustive list of search terms was developed. The exact search strategies used are presented in Appendix 1.

\section{Study records}

\section{Data management}

Results of the three electronic searches will be imported in EndNote X7.1 and merged. Duplicates will be identified and eliminated using an Excel form comparing authors' names and papers' titles and year of publication. A PDF document containing only title and abstract of each publication will be constituted and used for the first part of the selection process. Excel forms will be used to report acceptance or rejection of publications by reviewers and to compared results between reviewers. Once the selection process is over, additional Excel forms will be used for data extraction. Once data extraction is completed, all data will then be transferred into RevMan5.3. The members of the research team are all already experienced with the software and forms proposed.

\section{Selection process}

The reviewers will work independently and will be blinded regarding authors, journal, and year of publication of the papers. Two reviewers (V.W. and A.O.I.) will read each title and abstract and decide independently on retaining for further evaluation publications that potentially described or investigated a nonantibiotic approach for treatment or prevention of IMI at dryoff. At this stage, reviewers will be more liberal for inclusion of publications (i.e. in case of doubt, publication will be retained). The results of the review process of the reviewers will then be combined and, whenever the reviewers disagree, a third reviewer (D.F. or S.D.) will be asked to decide whether to retain the abstract for further evaluation. Full text of the publications selected at that point will be obtained, and reviewed in a similar fashion, but with strict application of the inclusions and exclusions criteria. 


\section{Data collection process}

Data for the trials will be extracted independently by three reviewers (V.W., D.F., and S.D.) in Excel forms. All the extracted data will then be compared between the three reviewers for completeness and accuracy.

\section{Data items}

The following information will be included: (1) study methods and characteristics: year of publication, country of study, funding, study design (i.e. randomized controlled trial (RCT), cohort, case-control, or meta-analysis), study characteristics (e.g. randomization and blindness for RCT, treatment of confounders for observational studies, use of natural or artificially induced IMI); (2) participants: number of herds, number of cows, number of quarter, age, breed, inclusion criteria; (3) intervention: type of non-antibiotic approach (i.e. biologics, homeopathy, botanicals, probiotics, other alternative products, vaccines, teat sealants), dose, route of administration and duration of treatment; (4) control groups: no treatment, placebo, or positive antibiotic control treatment (including dose, route of administration and duration of treatment); (5) results: types of outcome measured (i.e. IMI elimination or incidence rate, IMI prevalence at calving, or $\mathrm{CM}$ incidence) including diagnostic criteria (i.e. SCC, culture, or PCR threshold used to define IMI), reported outcomes, adverse events, follow-up time and results.

\section{Outcome and prioritization}

Primary outcomes are those previously mentioned: IMI elimination rate or post-calving IMI prevalence (for studies investigating IMI treatment); IMI incidence, post-calving IMI prevalence, or early lactation (i.e. 0-3 months) CM incidence (for studies investigating IMI prevention). Secondary outcomes that will be investigated are: subsequent lactation milk production, culling rate, and potential side-effects.

\section{Risk of bias in individual studies}

The author expects to mainly retrieve controlled trials. Assessment of risk of bias in included studies will, therefore, mainly be evaluated using the Cochrane Collaboration's tool for assessing risk of bias (Cochrane Handbook for Systematic Reviews of Interventions version 5.1.0) available through Review Manager (RevMan 5.3). It will include the appraisal of seven specific sources of bias commonly observed in clinical trials: random sequence generation and allocation concealment (two potential sources of selection bias); blinding of participants and personnel (a potential source of performance bias); blinding of outcome assessment (a potential source of detection bias); incomplete outcome data (a potential source of attrition bias); selective outcome reporting (a potential source of reporting bias); and, finally, any other potential bias not explicitly listed.
Risk of biases will first be assessed independently by three reviewers as 'low risk', 'high risk' or 'unclear risk'. Results of the three evaluations will then be compared and a consensus will be obtained in case of disagreement through discussion.

If publications using observational study designs are retained, then the Newcastle-Ottawa Scale (NOS) for assessing the quality of nonrandomized studies will be used to assess risk of bias for these specific studies (Wells et al.). The NOS tool was developed to judge studies using cohort or case-control study design on eight items categorized in three categories: (1) selection of the study groups; (2) comparability of the groups; and (3) ascertainment of exposure (case-control studies) or outcome (cohort studies) of interest. Bias assessment will otherwise be conducted as previously described (independent assessment by three reviewers and consensual decision in case of disagreement).

\section{Data synthesis and meta-bias}

Given that relatively homogenous data are available from a substantial number of studies on a given intervention, one or multiple meta-analyses will possibly be conducted. For each study, risk ratio $(R R)$ measures reporting risk of IMI elimination, risk of acquisition of new IMI, IMI risk post-calving, or risk of $\mathrm{CM}$ in the following lactation will be computed along with $95 \%$ confidence intervals. Heterogeneity between studies will be assessed using the $I^{2}$ statistic (Higgins and Green, 2011). If relevant, putative sources of heterogeneity (i.e. source of data used and IMI or CM case definition) will be investigated using subgroup analyses and univariate meta-regression. Metaregression will be conducted using the SAS 9.4 software PROC MIXED procedure (SAS Institute Inc., Cary, NC) as described by van Houwelingen et al. (2002). If relevant, an overall summary of effect will be computed using either a fixed or random effect model depending on the outcome at hand. Influence of each study will be investigated by deleting each study from the meta-analysis and determining change in the overall and subgroup summaries of effect as proposed by O'Connor et al. (2014). Risk of publication bias will then be investigated using funnel plots (Dohoo et al., 2009).

\section{Confidence in cumulative estimate}

If relevant, for each PICO questions, for each type of intervention reported, and for each outcome included, the authors will attempt to appraise the quality of evidence using the Grading of Recommendations Assessment, Development and Evaluation working group methodology (Guyatt et al., 2011).

\section{Acknowledgment}

The authors would like to acknowledge Rafael Rangel Braga, librarian, Faculté de Médecine Vétérinaire, Université de Montréal, for his precious help. 


\section{Contributions}

S.D. is the guarantor of the current review. D.F., V.W., A.O.I., and S.D. contributed to establishing the protocol for the systematic review. J.P.R., P.L., and F.L. provided their expertise on intramammary infections and organic dairy farming. All authors reviewed and provided feedback on the manuscript.

\section{Support}

This research is funded by the Agri-Innovation Program of Agriculture and Agri-Food Canada's Growing Forward 2 Policy Framework (a federal-provincial-territorial initiative), Dairy Farmers of Canada, and Valacta Inc through the Organic Science Cluster II, an industry-supported research and development endeavor initiated by the Organic Agriculture Centre of Canada at Dalhousie University in collaboration with the Organic Federation of Canada. The proposed research is also funded by one of the authors (SD) Natural Sciences and Engineering Research Council of Canada discovery grant. These organizations were not involved in any other aspects of the current research and will not contribute to the interpretation or publication of the results.

\section{References}

Bradley AJ and Green MJ (2000). A study of the incidence and significance of intramammary enterobacterial infections acquired during the dry period. Journal of Dairy Science 83: 1957-1965.

Bradley AJ and Green MJ (2004). The importance of the nonlactating period in the epidemiology of intramammary infection and strategies for prevention. Veterinary Clinics of North America - Food Animal Practice 20: 547-568.

Dohoo I, Martin W and Stryhn H (2009). Veterinary Epidemiologic Research. Charlottetown, PE, Canada: VER Inc.

Grindlay DJ, Brennan ML and Dean RS (2012). Searching the veterinary literature: a comparison of the coverage of veterinary journals by nine bibliographic databases. Journal of Veterinary Medical Education 39: 404-412.

Guyatt G, Oxman AD, Akl EA, Kunz R, Vist G, Brozek J, Norris S, Falck-Ytter Y, Glasziou P, Debeer H, Jaeschke R, Rind D, Meerpohl J, Dahm P and Schunemann HJ (2011). GRADE guidelines: 1. Introduction-GRADE evidence profiles and summary of findings tables. Journal of Clinical Epidemiology 64: 383-394.

Hektoen L, Larsen S, Odegaard SA and Loken T (2004). Comparison of homeopathy, placebo and antibiotic treatment of clinical mastitis in dairy cows - methodological issues and results from a randomizedclinical trial. Journal of Veterinary Medicine Series A 51: 439-446.

Higgins JPT and Green S (2011). Cochrane Handbook for Systematic Reviews of Interventions Version 5.1.0. Cambridge, UK: The Cochrane Collaboration.

Klostermann K, Crispie F, Flynn J, Ross RP, Hill C and Meaney W (2008). Intramammary infusion of a live culture of Lactococcus lactis for treatment of bovine mastitis: comparison with antibiotic treatment in field trials. Journal of Dairy Research 75: 365-373.

Kuipers A, Koops WJ and Wemmenhove H (2016). Antibiotic use in dairy herds in the Netherlands from 2005 to 2012. Journal of Dairy Science 99: 1632-1648.

Mathie RT and Clausen J (2014). Veterinary homeopathy: systematic review of medical conditions studied by randomised placebo-controlled trials. Veterinary Record 175: 373-381.
Mathie RT and Clausen J (2015). Veterinary homeopathy: systematic review of medical conditions studied by randomised trials controlled by other than placebo. BMC Veterinary Research 11: 236.

O'Connor AM, Sargeant JM and Wang C (2014). Conducting systematic reviews of intervention questions III: Synthesizing data from intervention studies using meta-analysis. Zoonoses and Public Health 61 (Suppl. 1): 52-63.

Pinedo P, Karreman H, Bothe H, Velez J and Risco C (2013). Efficacy of a botanical preparation for the intramammary treatment of clinical mastitis on an organic dairy farm. Canadian Veterinary Journal 54: 479-484.

Saini V, Mcclure JT, Léger D, Dufour S, Sheldon AG, Scholl DT and Barkema HW (2012). Antimicrobial use on Canadian dairy farms. Journal of Dairy Science 95: 1209-1221.

Van Houwelingen HC, Arends LR and Stijnen T (2002). Advanced methods in meta-analysis: multivariate approach and meta-regression. Statistics in Medicine 21: 589-624.

Wells G, Shea B, O'connell D, Peterson J, Welch V, Losos M and Tugwell P. The Newcastle-Ottawa Scale (NOS) for assessing the quality of nonrandomised studies in meta-analyses. [Available online at http:// www.ohri.ca/programs/clinical_epidemiology/oxford.asp] [Accessed July 12th 2016].

\section{Appendix 1. Boolean search strategies used in a systematic review of the literature on non-antibiotic approaches at drying-off for treating and preventing intramammary infections in dairy cows}

The search strategy for each database will be a combination of different components describing these four concepts of interest: disease (i.e. mastitis), population (i.e. dairy cows), period (i.e. dry period), and intervention (i.e. non-antibiotic treatment). Each component will include either key words (all databases), thesaurus terms (CAB Abstracts), or Medical Sub-Heading terms (MESH; Medline) describing the targeted concepts. Components are described below. Moreover, only articles published after 1969 will be retained.

\section{Search strategies}

\section{Web of Science}

Component 1 AND component 4 AND component 7 AND component 10 .

\section{$C A B$ Abstracts}

(Component 1 OR component 2) AND (component 4 OR component 5) AND (component 7 OR component 8) AND (component 10 OR component 11).

\section{Medline}

(Component 1 OR component 3) AND (component 4 OR component 6) AND (component 7 OR component 9) AND (component 10 OR component 12). 


\section{Components}

\section{Key words disease}

Subclinical mastitis OR Sub-clinical mastitis OR SCM OR Asymptomatic mastitis OR-Intramammary infection* OR Intra mammary infection* OR IMI OR Mastitis.

\section{CAB Abstracts thesaurus terms disease}

Exp mastitis/OR teat diseases

\section{Medline MESH terms disease}

Mastitis, Bovine/OR Staphylococcus aureus/OR Staphylococcal Infections/OR Streptococcal Infections/OR exp Cattle Diseases OR exp Mammary Glands OR exp Streptococcal Infections/OR exp Leukocyte Count.

\section{Key words population}

Cow* OR Cattle OR Bovine OR Dairy cow* OR Dried cow*.

\section{CAB Abstracts thesaurus terms population}

Cows/OR dairy cows.

\section{Medline MESH terms population}

Cattle/OR Animal.

\section{Key words period}

Dry-off OR Drying-off OR Dry period* OR Drying period* OR Drying-off period* OR Dry-off period*.

\section{CAB Abstracts thesaurus terms period}

Dry period.

\section{Medline MESH terms period}

Exp Postpartum Period/OR exp Lactation.

\section{Key words intervention}

Alternative treatments OR Approach* OR Method* OR Methodolog* OR Technique* OR Treatment* OR Prevention
OR Preventing OR Prevent OR Control OR Guideline* OR Protocol* OR Procedure* OR Alternative treatment* OR complementary treatment* OR alternative therap* OR complementary therap* OR alternative practice* OR alternative medicine* OR complementary medicine* OR botanical medicine* OR Chinese medicine* OR traditional medicine* OR traditional Chinese medicine* OR acupressure or acupuncture OR acutherapy OR anti-inflammatory $\mathrm{OR}$ antiinflammatory $\mathrm{OR}$ aromatherap* OR ascorbic acid OR aspirin OR bach flower OR bacteriocin* OR bacteriophage OR biocell* OR biologic* OR biological treatment* OR bismuth subnitrate OR chiroprati* OR clay OR endobacteria OR energy therap* OR essential oil* OR fatty acid* OR flunixin OR herbal drug* OR herbal medicine* OR herbal remed* OR homeopath* OR hydrotherap* OR hyperimmune serum OR immune serum OR immune stimula* OR immunobooster OR immunostimula* OR immunotherap* OR immuno modulatory OR immunization OR ketoprofen OR lactococcus lactis OR lantibiotic OR laser therap* OR medicinal plant* OR meloxicam OR natural products OR nonantibiotic* OR non antibiotic* OR non-steroidal anti-inflammatory agent* OR nsaid OR omega OR orbeseal OR organic* OR osteopath* OR phage OR physical therap* OR physiotherap* OR phytotherap* OR plant extract* OR platelet concentrate OR prebiotic* OR probiotic* OR teat seal* OR teat sealer* OR therapeutic massage* OR vitamin* OR vaccin*.

\section{$11 C A B$ abstract thesaurus terms intervention}

exp 'complementary and alternative medicine'/OR medicinal plants/OR herbal drugs/OR exp traditional medicines/OR exp traditional medicine/OR exp anti-inflammatory agents/ OR exp ascorbic acid/OR aspirin/OR bacteriocins/OR bacteriophages/OR biological treatment/OR bismuth/OR clay/OR exp plant oils/OR exp fatty acids/OR flunixin/OR herbal drugs/OR immune serum/OR immunostimulation/OR exp immunomodulators/OR ketoprofen/OR Lactococcus lactis/ OR lasers/OR natural products/OR exp non-steroidal antiinflammatory agents/OR exp bismuth/OR physical therapy/ OR exp plant products/OR blood products/OR platelets/OR seals/OR probiotics/OR prebiotics/OR massage/OR exp vitamins/OR immunization/OR vaccines OR vaccination.

\section{Medline MESH terms intervention}

exp Complementary Therapies/OR exp guideline/OR exp Clinical Protocols/OR exp Plant Extracts/OR exp Medicine, Chinese Traditional/OR exp Plants, Medicinal/OR exp Drugs, Chinese Herbal/OR exp Acupuncture Therapy/OR exp Phytotherapy/OR exp Medicine, East Asian Traditional/ OR exp Acupressure/OR exp Anti-Inflammatory Agents/OR exp Ascorbic Acid/OR exp Aspirin/OR exp Plant Extracts/ OR exp Bacteriocins/OR exp Bacteriophages/OR exp Bismuth/OR exp Chiropractic/OR exp Fatty Acids/OR exp Anti-Inflammatory Agents, Non-Steroidal/OR exp Clonixin/ 
OR exp Hydrotherapy/OR exp Immune Sera/OR exp Immunization/OR exp Ketoprofen/OR Lactococcus lactis/ OR exp Bacteriocins/OR Laser Therapy/OR Thiazines/OR Diclofenac/OR Thiazoles/OR Cyclooxygenase Inhibitors/OR Anti-Inflammatory Agents, Non-Steroidal/OR Cyclooxygenase 2/OR exp Anti-Inflammatory Agents, Non-Steroidal/OR exp Osteopathic Medicine/OR exp Platelet-Rich Plasma/OR exp Blood Preservation/OR exp Blood Transfusion/OR exp Blood Platelets/OR exp Thrombocytopenia/OR exp Platelet Transfusion/OR exp Platelet Count/OR exp Probiotics/OR exp Prebiotics/OR Massage/OR exp Vitamins/OR exp Vaccination/OR exp Vaccines. 